\title{
Review of: "American Robin (Turdus Migratorius) Blood Lead Levels May Reflect Elevated Soil Lead Levels: Further Consequences of the Flint Water Crisis"
}

\author{
Guillermo Espinosa-Reyes ${ }^{1}$ \\ 1 Universidad Autónoma de San Luis Potosí
}

Potential competing interests: The author(s) declared that no potential competing interests exist.

The central theme of the article is interesting, I consider that its sampling design is adequate and the writing of the document is good; however, I consider that some things are not clear in the methodology and the discussion carried out by the authors is por. For this reasons I consider that the article should not be accepted for publication.

- In the introduction the authors mention that lead exposure increases in humans and wildlife alike (Sordo and Casas 2006; Hanna-Attisha et al. 2016; Laidlaw et al. 2016). However, the exposure is different, generally animals are more exposed than humans, due to their behavioral and life habits (eg burrows of rodents, earthworms, etc).

- Additionally, we suggest that the authors add information on the advantages of using electrochemistry for the determination of trace elements and comparison with others, as well as a general description of the principle of the method used.

- In the methods, it is necessary to insert a map where the sampling points are clearly indicated.

- It remains to mention the methodology that was used to estimate the condition index and the variables that were used.

- It is important to specify the LeadCarell equipment model, as well as the procedure for the determination and for its calibration. Being a chemical analysis device, this is important information so that some other author can reproduce the methodology.

- The reason for making an adjustment of the $\mathrm{Pb}$ values measured by anodic extraction voltammetry (ASV) is not clear, when the same authors mention that the LeadCarell is a device that has proven to be exact, precise and highly correlated with other methods of blood lead analysis, such as graphite furnace atomic absorption spectrometry (GFAAS). We suggest that in the results section the authors place a table of the comparison of blood lead values with both methodologies. On the other hand, we suggest that the authors add the methodology or the procedure of the apparatus for the validation of the lead results.

- The authors must mention why they use a non-parametric test (KW) and then report the mean (Table 1 ). The correct way is to use the median as a measure of central tendency for nonparametric data. 
- In the discussion section the authors should add more information related to the effects of $\mathrm{Pb}$ in birds, since they make a lot of allusion to humans.

- We suggest making a comparative table where the results obtained in the present study are presented and comparing them with those reported in other articles already published (eg, the article by Chapa et al., 2010 needs to be cited).

- The authors should mention which is the area of activity of the american robins.

- The potential of robins as bioindicators is mentioned, but the advantages over other species that are more exposed compared to birds, such as earthworms, rodents, plant species, etc. are not mentioned. 